\title{
A Comparative Study of Transvaginal Sonography versus Laparoscopy Evaluation in Chronic Pelvic Pain: Original Study
}

\author{
Shugufta Y. Rather ${ }^{1}$, Sameena Sultana ${ }^{1}$, Sabia Rashid ${ }^{1}$, Imtiaz A. Wani ${ }^{2}$, Rauf A. Wani ${ }^{3}$ \\ ${ }^{1}$ Department of Obstetrics \& Gynecology, Lalla Ded Hospital, Govt. Medical College, Srinagar, Kashmir, India \\ ${ }^{2}$ Department of Surgery, Directorate of Health Services, Kashmir, India \\ ${ }^{3}$ Department of Surgery, Sheri Kashmir Institute of Medical Sciences, Srinagar, Kashmir, India
}

Email address:

imtazwani@gmail.com (I. A. Wani)

\section{To cite this article:}

Shugufta Y. Rather, Sameena Sultana, Sabia Rashid, Imtiaz A. Wani, Rauf A. Wani. A Comparative Study of Transvaginal Sonography versus Laparoscopy Evaluation in Chronic Pelvic Pain: Original Study. International Journal of Clinical and Experimental Medical Sciences.

Vol. 1, No. 1, 2015, pp. 4-6. doi: 10.11648/j.ijcems.20150101.12

\begin{abstract}
Introduction: Chronic pelvic pain (CPP) is intermittent or constant pain in the lower abdomen or pelvis for at least six months duration, not occurring exclusively with menstruation or intercourse and not associated with pregnancy. This chronic pelvic pain (CPP) is poorly understood, and may have significant impact on a woman's health. Transvaginal sonography (TVS) and laparoscopy are useful in the evaluation of women with CPP. This research was designed to study role of TVS and diagnostic laparoscopy in chronic pelvic pain. Materials and Methods: This prospective study was done at Lalla Ded Hospital from August 2011 to July 2014. All female patients who had clinical diagnosis of CPP were evaluated with the TVS and laparoscopy and findings were recorded. Results: Total of 80 women had CPP. Normal TVS was present in 41 cases $(51.25 \%)$. The remaining 39 (48.75\%) had an abnormal scan. Laparoscopic confirmation of pelvic pathology was reported in 40 cases with abnormal scan but normal scan was confirmed only in $10(12.50 \%)$ cases. Conclusion: In spite of similar specificity and positive predictive value, laparoscopy although being an invasive procedure still has got distinct advantages over TVS in terms of sensitivity and negative predictive value indicating its superiority in management.
\end{abstract}

Keywords: Chronic, Pelvic, Pain, TVS, Laparoscopy

\section{Introduction}

Chronic pelvic pain (CPP) is defined as pain of at least 6 months duration that occurs in the lower abdomen or below the umbilicus and has resulted in functional or psychological disability or required intervention and treatment [1]. The causes are often obscure and patients of the CPP have significant disruption of their quality of life and nature varies from constant to recurring [2]. Chronic pelvic pain is one of the most frustrating and controversial areas of gynecological practice [3]. The pelvic examinations are normal except a vague tenderness and commonly a physician diagnoses pelvic inflammatory disease. As a result, the women are given repeated course of antibiotics and often labeled as neuro-psychotics [4]. Careful history taking, meticulous examination and relevant investigation are three cornerstones for evaluating a patient with CPP [5].Laparoscopy is an effective tool in the evaluation of women with CPP [6]. The aim of this research is studying role of TVS and diagnostic laparoscopy in chronic pelvic pain.

\section{Materials and Methods}

This prospective study was done at Lalla Ded Hospital from August 2011 to July 2014. The study was approved by ethical committee of hospital. Written consent was taken after explaining procedures to patients. All patients who had diagnosis of CPP were subjected to detailed history and clinical examination. Women with known chronic medical or gynecologic diseases or any pelvic surgeries that were associated with lower abdominal pain were excluded from this study. All cases that had not gynecological cause were excluded by abdominal ultrasonography. While recording the history, particular enquiry was made regarding associated symptoms like dysmenorrhea, abnormal vaginal bleeding, vaginal discharge, dyspareunia, infertility, enteric symptoms, urological and musculoskeletal symptoms. Routine 
investigations were done in all. All women were subjected to high frequency TVS evaluation by experienced sonographer. TVS was done in postmenstrual phase. A standard technique of diagnostic laparoscopy was done by $10 \mathrm{~mm}$ port at infraumbilical area for 30-degree telescope and another one by two $5 \mathrm{mmipsilateral} \mathrm{working} \mathrm{ports.} \mathrm{The} \mathrm{interval} \mathrm{between} \mathrm{TVS}$ examination and laparoscopy ranged from 0 day to 15 days. The findings of TVS and diagnostic laparoscopy in each patient were recorded.

The data was analyzed by using Statistical Package for Social Science (SPSS) version 16 (SPSS Inc., USA). Qualitative data, quantitative data, frequency, mean, standard deviation (SD), and percent distribution were calculated. Chi square test was used for comparison between groups. For interpretation of results, $p$ value $<0.05$ was considered significant.

\section{Results}

Total of 80 women had CPP after excluding all nongynecological causes. All had transvaginal sonography and laparoscopic evaluation. Age ranged from 17-50 years with nullipara showing maximum number of $48(60 \%)$ cases. (Table 1\& 2) Associated symptoms like dysmenorrhea, menstrual irregularities, dyspareunia and infertility were also presented in $80 \%$ of cases. Normal pelvic examination was reported in $70(87.50 \%)$ patients except for mild tenderness which was considered normal. 5 patients had bulky uterus and had restricted mobility and tenderness in per vaginal examination. Adhesions were detected in 9 cases (11.25\%) and pelvic congestion in 6 cases $(7.5 \%)$ by laparoscopy only.

Table 1. Age incidence.

\begin{tabular}{lll}
\hline AGE(YEARS) & No. OF PATIENTS & $\%$ \\
\hline $17-20$ & 18 & 22.50 \\
$21-30$ & 42 & 52.50 \\
$31-40$ & 14 & 17.50 \\
$41-50$ & 6 & 7.50 \\
\hline
\end{tabular}

Table 2. Parity wise distribution.

\begin{tabular}{lll}
\hline Parity & Number & \% \\
\hline Po & 48 & 60 \\
P1 & 20 & 25 \\
P2 & 8 & 10 \\
P3\& above( all were ligated) & 4 & 5 \\
\hline
\end{tabular}

Table 3. Comparison of findings by TVS \& laparoscopy.

\begin{tabular}{lll}
\hline FINDINGS & TVS & LAPROSCOPY \\
\hline Normal & $41(51.25 \%)$ & $10(12.50 \%)$ \\
Ovarian cyst & $10(12.50 \%)$ & $12(15 \%)$ \\
TO mass & $6(7.5 \%)$ & $7(8.75 \%)$ \\
Chronic PID & $9(11.25 \%)$ & $15(18.75 \%)$ \\
Endometriosis & $8(10 \%)$ & $13(16.25 \%)$ \\
Tubercular lesion & Nil & $2(2.5 \%)$ \\
Pelvic congestion & Nil & $6(7.5 \%)$ \\
Fibroid uterus & $3(3.75 \%)$ & $2(2.5 \%)$ \\
Adhesions & Nil & $9(11.25 \%)$ \\
Bulky uterus with adenomyosis & $3(3.75 \%)$ & $4(5 \%)$ \\
\hline
\end{tabular}

TVS: Trans-vaginal sonography, TO: Tubo-ovarian, PID: Pelvic inflammatory disease

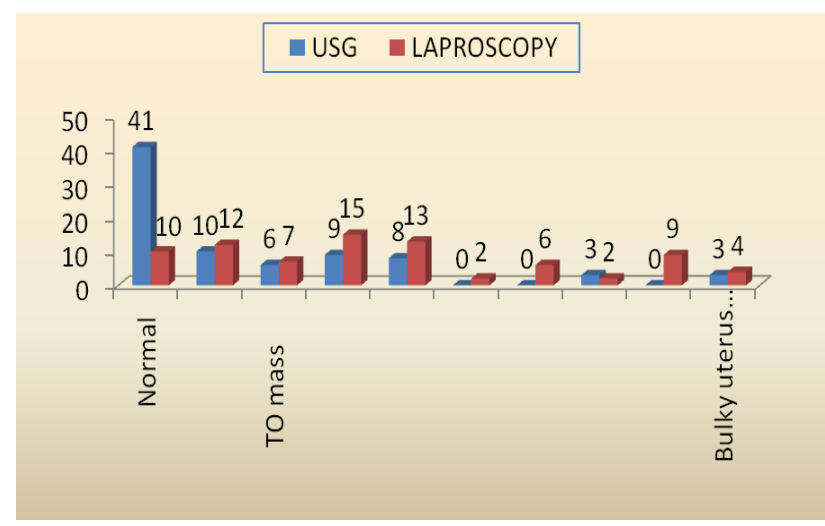

Figure 1. Graphic representation of Comparison of findings by TVS (USG) \& laparoscopy.

Normal TVS was reported in $41(51.25 \%)$ cases. The remaining $39(48.75 \%)$ cases had abnormal scan. Laparoscopic confirmation of pelvic pathology was reported in 40 cases with abnormal scan but normal scan was confirmed only in $10(12.50 \%)$ cases. 30 cases with normal scan had abnormal laparoscopy findings which were statistically significant, the majority had adhesions, salpingitis, endometriosis and tubercular lesions (Table 3).

Sensitivity and specificity for TVS were $58.57 \%$ and $100 \%$ respectively, while for laparoscopy it was $98.57 \%$ sensitivity and $100 \%$ specificity. Positive predictive value was $100 \%$ by both means but negative predictive value was significantly higher by laparoscopy rather than TVS; $90 \%$ compared to $24.34 \%$.

\section{Discussion}

Chronic pelvic pain is a common and debilitating condition; its etiology is multifactorial, involving social, psychological and biological factors [1]. Chronic pelvic pain (CPP) is one of the commonest symptomatology in gynecological outpatient clinics. It accounts for $10 \%$ of office visits to gynecologists and general clinics and prevalence of $3.8 \%[4,7]$. In the present study the maximum number of women with CPP belonged to age group of 21-40years old. This is similar to findings of Goswami et al., Kamilya et al., and Veena et al. [8, 9, and 10]. Clinical examination did not detect any abnormalities in $70(87.50 \%)$ patients.

TVS was normal in $41(51.25 \%)$ women whereas on laparoscopy no detectable pathology was detected in 10 $(12.5 \%)$ women. Laparoscopy is more sensitive in detecting chronic pelvic pain especially mild endometriosis, pelvic inflammatory diseases (PID) and adhesions. Laparoscopy is an excellent tool in evaluation of patients with pelvic pain, because diagnosis and often treatment can be accomplished in one sitting, without subjecting the patients to exploratory laparotomy [6]. Negative laparoscopic findings were reported in literatures vary from 5\%-90\% $[9,10,11]$.

In our study adhesions were detected in $11.25 \%$ of patients which were due to pelvic inflammatory diseases, postoperative or endometriosis in origin. Adhesions were most frequent findings in studies of Goswamiet al. (34.1\%), Mara 
et al. (22.3\%), Krech et al. (38\%) and Newham (40\%) [8 and 12-14].

PID demonstrated by presence of salpingitis, tuboovarian mass, hydrosalpinx fluid, pus in pouch of Douglas (POD) or obliteration of POD was detected in $18.75 \%$ patients in comparison to $30.3 \%$ by Goswami et al. and $17.7 \%$ by Mara et al. [8, 12]. Endometriosis was more commonly found in $16.25 \%$ in our study compared to $5 \%$ as reported by Veena Agarwal and $13.9 \%$ by Goswami, Sebantiet al. [8, 10]. Pelvic congestion was seen in $75 \%$ cases. Howard reported that more than $40 \%$ of laparoscopies are performed for diagnosis of CPP [15]. About 75\% of patients of CPP with normal TVS findings had at least one detectable diagnosis by laparoscopy.

But because of invasive nature and cost, decision to perform a laparoscopy should be reserved and taken after a proper history, physical examination, findings of noninvasive test, after exclusion of non-gynecological causes of pelvic pain and after failure of conservative treatment. Also because few women who underwent laparoscopy for reasons other than investigation of pain, had comparable findings that were not associated with pain symptoms.

\section{Conclusion}

Laparoscopy may be considered as a gold standard in the evaluation of CPP. Laparoscopy although being an invasive procedure, still has got distinct advantages over TVS in terms of sensitivity and negative predictive value indicating its superiority in management.

\section{References}

[1] Cheong YC, Smotra G, Williams AC. Non-surgical interventions for the management of chronic pelvic pain. Cochrane Database Syst Rev. 2014 Mar 5; 3: CD008797

[2] Pierce AN, Christianson JA. Stress and chronic pelvic pain. ProgMolBiolTransl Sci. 2015; 131: 509-35.
[3] Soper DE. What is new in the mechanisms of chronic pelvic pain? best articles from the past year. Obstet Gynecol. 2015 Mar; 125(3): 729-31.

[4] R.W Beard, E. M Belsey, B.A Liberman, J.C.M Wilkinson. Pelvic pain in women. Am J Obstet Gynecol 1997(1); 128: 566-570.

[5] Amirbekian S, Hooley RJ. Ultrasound evaluation of pelvic pain. Radiol Clin North Am. 2014; 52(6): 1215-35.

[6] Shripad H, Chander C. Role of laparoscopy in evaluation of chronic pelvic pain. J Minim Access Surg. 2005; 1(3): 116120.

[7] Stout AL, Steege JF, Dodson WC, Hughes CL. Relationship of laparoscopic findings to self-report of pelvic pain. Am J Obstet Gynecol. 1991 Jan; 164(1 Pt 1): 73-9.

[8] Goswami S, Chakraborty P S, Datta R. Laparoscopy in chronic pelvic pain. J Obstet Gynecol India. 2008; 58(5): 435437.

[9] Howard FM. Chronic Pelvic Pain. Obstet Gynecol 2003; 101: 594-611.

[10] Kamilya G, Mukherji J, Gayen A. Different methods for evaluation of chronic pelvic pain. J Obstet Gynecol India 2005; 55: $251-3$

[11] Veena Agrawal and Roli Gautam. Is Laparoscopy necessary in all cases of chronic pelvic pain? Asian journal of Obs Gynae practice 2004; 89(3): 12- 14 .

[12] Mara M, Fucikova Z Kuzei D. Laparoscopy in chronic pelvic pain, a retrospective clinical study. Ceska Gynecol 2002; 67: $38-46$.

[13] Kresch AJ, Seifer DB, Sachs LB. Laparoscopy in 100 women with chronic pelvic pain. Obstet Gynecol 1984; 64: 672-4.

[14] Newham AP, van der Spuy ZM, Nugent F. Laparoscopic findings in women with chronic pelvic pain. S Afr Med J 1996; 86: 1200-3.

[15] Howard FM. The role of laparoscopy as a diagnostic tool in chronic pelvic pain. Ballivres best pract. Res, Clin.Gynecol 2000; 14 (3): 467-494. 